\title{
Effects of Lactobacillus kefiranofaciens M1 isolated from kefir grains on enterohemorrhagic Escherichia coli infection using mouse and intestinal cell models
}

\author{
Y. P. Chen, ${ }^{*}$ T. Y. Lee, ${ }^{*}$ W. S. Hong, ${ }^{*}$ H. H. Hsieh, ${ }^{*}$ and M. J. Chen ${ }^{*}{ }^{1}$ \\ *Department of Animal Science and Technology, and \\ †Center for Biotechnology, National Taiwan University, Taipei 10672, Taiwan (ROC)
}

\begin{abstract}
A potential probiotic strain, Lactobacillus kefiranofaciens M1, was previously isolated from kefir grains, which are used to manufacture the traditional fermented drink kefir. The aim of this study was to investigate the effects of $L b$. kefiranofaciens M1 on enterohemorrhagic Escherichia coli (EHEC) infection, using mice and intestinal cell models. BALB/c mice were daily administrated with either phosphate buffered saline or Lb. kefiranofaciens M1 at $2 \times 10^{8} \mathrm{cfu} /$ mouse per day intragastrically for $7 \mathrm{~d}$. Intragastric challenges with EHEC $\left(2 \times 10^{9} \mathrm{cfu} /\right.$ mouse $)$ were conducted on d 0, 4, and 7 after treatment. Administration of Lb. kefiranofaciens M1 was able to prevent EHEC infection-induced symptoms, intestinal damage, renal damage, bacterial translocation, and Shiga toxin penetration. Furthermore, the mucosal EHEC-specific IgA responses were increased after $L b$. kefiranofaciens M1 administration in the EHEC-infected mouse system. Additionally, in vitro, Lb. kefiranofaciens M1 was shown to have a protective effect on Caco-2 intestinal epithelial cells and Caco-2 intestinal epithelial cell monolayers; the bacteria limited EHEC-induced cell death and reduced the loss of epithelial integrity. These findings support the potential of $L b$. kefiranofaciens M1 treatment as an approach to preventing EHEC infection and its effects.
\end{abstract}

Key words: probiotic, Lactobacillus kefiranofaciens M1, kefir, enterohemorrhagic Escherichia coli

\section{INTRODUCTION}

Outbreaks of enterohemorrhagic Escherichia coli (EHEC) infection are a severe epidemiological problem worldwide. By 2011, authorities have reported 22 fatalities and ca. 2,000 infections related to EHEC, with steadily increasing numbers. The transmission

Received May 9, 2013.

Accepted August 31, 2013.

${ }^{1}$ Corresponding author: cmj@ntu.edu.tw routes of EHEC are associated with the ingestion of contaminated food, including beef, vegetables, fruit, and water (Serna and Boedeker, 2008; Mohawk and O'Brien, 2011). Particularly, EHEC serotype O157:H7 has been found to be involved in many large-scale foodborne infectious disease outbreaks recently (Lim et al., 2010). Enterohemorrhagic E. coli are defined as strains of $E$. coli that cause hemorrhagic colitis and possess the ability to produce Shiga toxin (Stx), which can damage intestinal epithelial cells directly (Kaper et al., 2004). Shiga toxins are also able to attack renal endothelial cells and induce hemolytic uremic syndrome (Hodges and Gill, 2010; Lim et al., 2010), which may cause acute renal failure in humans (Tarr et al., 2005). No effective treatment for EHEC infection exists yet. Only supportive treatments, such as intravenous supplementation with saline or isotonic crystalloid (Tarr et al., 2005), can be provided clinically. Although several novel strategies have been proposed for the treatment of EHEC infection, including the application of antibiotics, the use of Stx-binding agents, treatment with antithrombotic agents, and vaccination, none of them have been successfully applied in the field due to their limitations in terms of clinical practice. Thus, searching for an efficient way to prevent or ameliorate EHEC infection is urgently needed (Tarr et al., 2005; Serna and Boedeker, 2008).

Probiotics are live microbes that are able to improve human health, including the amelioration of gastrointestinal disorders. Certain species or strains of probiotics have been shown to have antiinfective or antibacterial ability against various intestinal pathogens in vitro and in murine models via various putative mechanisms (Gareau et al., 2010). Among these strains, several lactobacilli and bifidobacteria have been shown to have potential in preventing EHEC infection and when treating disease caused by EHEC (DuPont and DuPont, 2011). It has been suggested that the possible mechanism by which these bacteria act against EHEC may involve alterations in the intestinal microbiota after consumption of the probiotic strain (DuPont and DuPont, 2011). However, no direct evidence has shown 
why alternation of microbiota by administration of probiotics could reduce EHEC infection.

Kefir, which is made by inoculating kefir grains into milk, is an alcoholic fermented dairy drink that has multiple health-promoting properties (Guzel-Seydim et al., 2011). The microorganisms presenting in kefir grains, mainly consisting of lactic acid bacteria and yeasts, might play a key role in its functionalities. The composition of kefir grains is influenced by many environmental factors (Chen et al., 2008; Wang et al., 2008; Guzel-Seydim et al., 2011). Santos et al. (2003) indicated that some Lactobacillus spp. isolated from European kefir grains show in vitro antimicrobial activity against E. coli. However, the inhibition and prevention of EHEC or other pathogenic E. coli infection in vivo by kefir itself or by any microbe isolated from kefir grains has not been reported.

In our previous study, several potential probiotic strains were isolated from Taiwanese kefir grain (Chen et al., 2008). Among these strains, Lactobacillus kefiranofaciens M1 came to our attention. This strain has been shown to demonstrate effective immunomodulating, antiallergic effects, and antiasthmatic activity, both in vitro and in vivo (Hong et al., 2009, 2010, 2011). It is worth noting that the heat-killed $L b$. kefiranofaciens M1 also demonstrated strong antiallergic effects. Most recently, we have also shown that $L b$. kefiranofaciens M1 is able to strengthen the intestinal barrier and prevent chemical-induced colitis in a dextran sodium sulfate (DSS)-mouse model and that it does this via a tolllike receptor (TLR)2-dependent pathway (Chen et al., 2012; Zhang et al., 2012). We further analyzed the cell wall composition and found that Lb. kefiranofaciens M1 possessed glycosyltransferase (GlcNAc; 64\%), glucose $(26 \%)$, and galactose (10\%), which was very different from type strain Lb. kefiranofaciens BCRC 16059 (our unpublished data). According to these findings, $L b$. kefiranofaciens M1 is a unique strain. These features make $L b$. kefiranofaciens M1 a perfect candidate probiotic bacterium for protecting against enteric pathogen infection. Therefore, the objective of this study was to investigate the efficacy of $L b$. kefiranofaciens M1 on a preventive measure against EHEC O157:H7 infection using an in vivo mouse and an in vitro cell model. This study might also provide the scientific evidence explaining the certain functionalities involving in kefir milk.

\section{MATERIALS AND METHODS}

\section{Lactobacillus kefiranofaciens M1 Sample Preparation}

Lactobacillus kefiranofaciens M1 was isolated and identified previously (Chen et al., 2008). Lactobacillus kefiranofaciens M1 was cultured in de Man, Rogosa, and Sharp (MRS) broth (Difco Laboratories Inc., Detroit, MI) at $37^{\circ} \mathrm{C}$ and was harvested during log phase by washing and resuspending 3 times in PBS (HyClone Laboratories Inc., South Logan, UT). After washing, the bacterial cells were resuspended in PBS and adjusted to the indicated concentrations. Heat-inactivated Lb. kefiranofaciens $\mathrm{M} 1$ was prepared by heating at $85^{\circ} \mathrm{C}$ for $40 \mathrm{~min}$ as described previously (Hong et al., 2011).

\section{EHEC 0157:H7 Preparation}

Enterohemorrhagic E. coli O157:H7 ATCC 35150 was obtained from the American Type Culture Collection (Manassas, VA). Enterohemorrhagic E. coli O157:H7 was cultured in tryptic soy broth (TSB; Acumedia, Lansing, MI) at $37^{\circ} \mathrm{C}$ for $12 \mathrm{~h}$ and was harvested by washing and resuspending 3 times in PBS (HyClone Laboratories Inc.) to the indicated concentrations.

\section{EHEC Infection Model}

The EHEC infection scheme in mice was modified from the one published by Mohawk et al. (2010). Eightweek-old specific pathogen-free female BALB/c mice (National Laboratory Animal Center, Taipei, Taiwan) were maintained in a standard cage environment at 23 to $25^{\circ} \mathrm{C}$ while being exposed to a 12 -h light and dark cycle. All experiments were approved by Institutional Animal Care and Use Committee of National Taiwan University (Taipei, Taiwan) and performed in accordance with guidelines for animal care of the National Science Council in Taipei, Taiwan (IACUC approval number NTU-100-EL-100). Mice with similar BW were separated to give 6 to 9 mice per group. These groups were administrated daily with either PBS (HyClone Laboratories Inc.) or $2 \times 10^{8}$ cfu of Lb. kefiranofaciens M1/mouse per day intragastrically for $7 \mathrm{~d}$. This was followed by intragastric challenges with EHEC O157:H7 $\left(2 \times 10^{9} \mathrm{cfu} /\right.$ mouse $)$, which were conducted at d 0,4 , and 7 after the end of Lb. kefiranofaciens M1 treatment. Food intake was recorded during the infection period. The mice were killed at d 9 by cervical dislocation, which was followed by organ collection. The weights of the cecum, spleen, and kidneys were recorded.

\section{Fecal Bleeding Assessment}

The feces were collected from the mice and immediately the amount of occult blood in feces was measured using a Hemoccult Sensa assay (Beckman Coulter Inc., Brea, CA; Zhang et al., 2012).

\section{Analysis of EHEC 0157:H7 Amount in Organs and Blood}

Liver, spleen, and blood samples were collected using an aseptic procedure in a laminar flow cabinet. Blood 
samples were collected by submandibular bleeding and cultured in brain heart infusion (BHI; Acumedia) overnight and then plated on EHEC O157-selective agar CHROMagar O157 (CHROMagar, Paris, France) for colony enumeration. Alternatively, tissue samples from the various organs were homogenized in $0.1 \%$ peptone water using a gentleMACS Dissociator (Miltenyi Biotec GmbH, Bergisch Gladbach, Germany) and then plated on EHEC O157 selective agar CHROMagar O157 (CHROMagar) for colony enumeration.

\section{Histological Evaluation}

Histological changes affecting the distal ileum, as well as the colon, cecum, and kidney, were observed after hematoxylin and eosin staining. The feces in the intestines and the contents of the cecum were removed by flushing with ice-cold PBS (HyClone Laboratories Inc.). Intestinal fragments that were $1 \mathrm{~cm}$ long were isolated and then washed with ice-cold PBS (HyClone Laboratories Inc.). The washed intestinal fragments and intact kidneys were immediately immersed in 10\% histological grade phosphate-buffered formalin (Mallinckrodt Chemical Ltd., Derbyshire, UK) for fixation. The fixed tissue and organ samples were dehydrated in ethanol and then embedded in paraffin wax, which was followed by sectioning (5- $\mu \mathrm{m}$ thickness) and staining with hematoxylin and eosin stain. Histological evaluation was carried out using the intestine scoring system developed by Dieleman et al. (1998) and each sample was evaluated by well-trained histologists. Briefly, the histological score of each intestinal tissue section was based on the level of tissue inflammation, the extent of tissue involved in the inflammation, the amount of tissue regeneration present, and the amount of crypt damage that had occurred.

\section{Measurement of Immunoglobulin Production}

The levels of total IgG, IgM, and IgA in the feces and blood were measured using mouse $\operatorname{IgG}, \operatorname{IgM}$, and IgA ELISA quantification kits (Bethyl Laboratories, Montgomery, TX), respectively. Enzyme-linked immunosorbent assays were performed using the standard protocol as recommended by the manufacturer. The levels of anti-E. coli O157:H7-specific IgG, IgM, and IgA in sera or feces were also measured using an ELISA-based method (Shu and Gill, 2002; Gagnon et al., 2006). Briefly, formalin-killed EHEC O157:H7 (5 $\times 10^{6}$ cells) was coated onto a MaxiSorp microplate (Nunc, Thermo Fisher Scientific, Waltham, MA) in 100 $\mu \mathrm{L}$ of PBS $(\mathrm{pH} 7.3)$ at $4^{\circ} \mathrm{C}$ overnight. After coating, the plates were washed 4 times with $0.1 \%$ Tween 20 in PBS (PBST) and then blocked by blocking buffer $(0.1 \%$ Tween 20 and $3 \%$ BSA in PBS) for $1 \mathrm{~h}$ at room temperature. Supernatants obtained from the various fecal suspensions $(100 \mathrm{mg} / \mathrm{mL})$ in dilution buffer $(0.5 \%$ BSA in PBST) and sera obtained from the blood samples were added to the coated wells and incubated for $2 \mathrm{~h}$ at room temperature. After incubation, the plates were washed 4 times with PBST. This was followed by adding biotinylated anti-mouse IgG, IgM, or IgA antibodies (Bethyl Laboratories) for $2 \mathrm{~h}$ at room temperature. The plates were then washed 4 times with PBST, which was followed by treatment with streptavidin-horseradish peroxidase (BioSource, Invitrogen Corp., Carlsbad, CA) for $30 \mathrm{~min}$ at room temperature. Next, the plates were washed again and then finally treated with $3,3^{\prime}, 5,5^{\prime}$-tetramethylbenzidine (Clinical Science Products Inc., Mansfield, MA) for 30 min at room temperature. This reaction was stopped by adding $1 \mathrm{~N} \mathrm{H}_{2} \mathrm{SO}_{4}$. The results were collected by measuring absorbance at $450 \mathrm{~nm}$ using an ELISA microplate reader (VMax; Molecular Devices LLC, Sunnyvale, CA).

\section{Measurement of Stx Production}

The level of Stx-1 and Stx-2 in the sera and fecal supernatants $(100 \mathrm{mg} / \mathrm{mL})$ were measured using a Prolisa EHEC EIA assay kit (Pro-Lab Diagnostics, Toronto, ON, Canada) according to the manufacturer's instructions. The level of Stx in the EHEC cultured medium was measured after 10-fold dilution in deionized distilled $\mathrm{H}_{2} \mathrm{O}$.

\section{Assessment of Intestinal Epithelial Cell Viability}

Human colonic epithelial cell line Caco2-C2BBe1 was obtained from Bioresource Collection and Research Center (Hsinchu, Taiwan) and cultured in Dulbecco's modified Eagle's medium (HyClone Laboratories Inc.) supplemented with $10 \%$ heat-inactivated fetal bovine serum (Invitrogen Corp.), $50 \mu \mathrm{g} / \mathrm{mL}$ of penicillin, 50 $\mu \mathrm{g} / \mathrm{mL}$ of streptomycin sulfate, and $100 \mu \mathrm{g} / \mathrm{mL}$ of neomycin sulfate (Invitrogen Corp.) in a humidified atmosphere of $5 \% \mathrm{CO}_{2}$ at $37^{\circ} \mathrm{C}$. For the cell viability test, Caco- 2 cells were seeded onto a 96 -well plate $\left(10^{4}\right.$ cells/well) and cultured overnight. After that, the cells were treated with $L b$. kefiranofaciens M1 at indicated concentrations $\left(2 \times 10^{4}\right.$ or $10^{5} \mathrm{cfu} /$ well $)$ for $24 \mathrm{~h}$, which was followed by challenging with EHEC $\left(10^{4} \mathrm{cfu} /\right.$ well $)$ for $24 \mathrm{~h}$. For the lactose dehydrogenase (LDH) assay (BioVision Inc., Milpitas, CA), the cell medium was collected after treatment and filtered using a $0.22-\mu \mathrm{m}-$ pore-size membrane (Pall Corp., Port Washington, 
NY); the sample was then analyzed to obtain cell viability according to the manufacturer's instructions. For the 3-(4,5-dimethyl-2-thiazolyl)-2,5-diphenyl-2Htetrazolium bromide (MTT; Sigma, St. Louis, MO) assay, the remaining cells were washed with $100 \mu \mathrm{L}$ of PBS (HyClone Laboratories Inc.) 3 times and then incubated with $1 \mathrm{mg} / \mathrm{mL}$ of MTT in cell medium for $2 \mathrm{~h}$ at $37^{\circ} \mathrm{C}$ in a $5 \% \mathrm{CO}_{2}$ incubator; at the end of $2 \mathrm{~h}$, the MTT medium was removed and $150 \mu \mathrm{L}$ of dimethyl sulfoxide (Sigma) was added to each well to dissolve and resuspend the formazan crystals. Finally, the optical density (OD) at $570-\mathrm{nm}$ wavelength was measured. The OD value of the nontreated control group was used to indicate $100 \%$ cell viability. The cell viabilities (\%) of the other groups were calculated by dividing their OD values by the OD value of the nontreated control group.

\section{Caco-2 Intestinal Epithelial Cell Monolayer Preparation}

Caco-2 cells were seeded onto permeable 12-well Transwell membranes (Corning, Lowell, MA) with a $3-\mu \mathrm{m}$ pore size (cell density of $10^{5} / \mathrm{cm}^{2}$ ) for $14 \mathrm{~d}$. The culture medium was replaced with fresh medium every $2 \mathrm{~d}$ during the culture period. After $14 \mathrm{~d}$, the transepithelial electrical resistance (TEER) was measured using an EVOM Epithelial Voltohmmeter with an STX2 probe (World Precision Instruments, Sarasota, FL). The Caco-2 epithelial monolayers were ready to use when their TEER values were greater than $300 \Omega \mathrm{cm}^{2}$ (Zhang et al., 2012).

\section{Measurement of Caco-2 Cell Monolayer Integrity}

The Caco-2 monolayers were cocultured with $10^{7} \mathrm{cfu}$ of Lb. kefiranofaciens $\mathrm{M} 1$ at $37^{\circ} \mathrm{C}$ for $24 \mathrm{~h}$, which was followed by the addition of $10^{6} \mathrm{cfu}$ of EHEC or EHEC supernatant $(20 \% \mathrm{vol} / \mathrm{vol})$ for $12 \mathrm{~h}$ (Johnson-Henry et al., 2008). Transepithelial electric resistance values before coculture with $L b$. kefiranofaciens M1 and after treatment with EHEC were measured. The TEER change (\%) was expressed as the ratio of the TEER after EHEC treatment to the initial value for each experiment group (Zhang et al., 2012).

\section{Statistical Analysis}

Values are given as mean \pm standard deviation. All results were analyzed by Student's $t$-test or one-way ANOVA followed by Duncan's multiple range test using SAS software (SAS Institute Inc., Cary, NC). A $P$-value of $<0.05$ was indicative of a significant difference.

\section{RESULTS}

\section{Administration of Lb. kefiranofaciens M1 Ameliorates the Symptoms of EHEC Infection}

First, we examined the effect of orally administered Lb. kefiranofaciens M1 on EHEC O157:H7 challenge using a mouse model. The feed intake per BW was decreased in the EHEC infection group compared with the nontreated control group. Pretreatment with $L b$. kefiranofaciens M1 was able to prevent the decrease in feed intake caused by EHEC infection (Figure 1A). Administration of Lb. kefiranofaciens M1 was also able to improve the symptoms of EHEC infection-induced hemolytic colitis. The fecal occult blood tests showed that 5 out of 9 of the mice $(55.56 \%)$ in the EHEC infection group had fecal occult blood present, whereas only 1 out of $9(11.11 \%)$ were found to have fecal occult blood present in the Lb. kefiranofaciens M1 pretreatment group. Additionally, the increase in cecum weight and the decrease in kidney weight induced by EHEC infection were both restored in the Lb. kefiranofaciens M1 pretreatment group; however, no such restoration of the increase in spleen weight was observed (Figure 1B).

\section{Administration of Lb. kefiranofaciens M1 Prevents Intestinal Architecture Damage and Reduces Renal Injury Induced by EHEC Infection}

The tissue sections of the distal ileum, cecum, and proximal colon were evaluated by histological examination. We observed that the mice pretreated with $L b$. kefiranofaciens M1 showed a restoration of the atrophy of the microvilli. Furthermore, an amelioration of the loss of crypt and Paneth cells caused by the EHEC infection in distal ileum was also apparent (Figure 2). Enterohemorrhagic E. coli-induced tissue edema and loss of epithelial integrity in the cecum (Figure 3A; Supplemental Figure S1, available online at http:// dx.doi.org/10.3168/jds.2013-7015) and the proximal colon (Figure 3B; Supplemental Figure S2) were also reduced by $L b$. kefiranofaciens M1 pretreatment. The results were parallel to the qualitative observations outlined above. Pretreatment with Lb. kefiranofaciens M1 was found to significantly prevent the cecum and colon tissue damage that was induced by EHEC infection in the mice (Figure 3).

Additionally, severe congestion of the renal glomeruli and kidney interstitial tissue, which was found to be present in the EHEC-infected mice, was found to be largely absent in the $L b$. kefiranofaciens M1-pretreated rats. Only slight congestion of these tissues (Figure 4) was found, which means that the treatment was able 


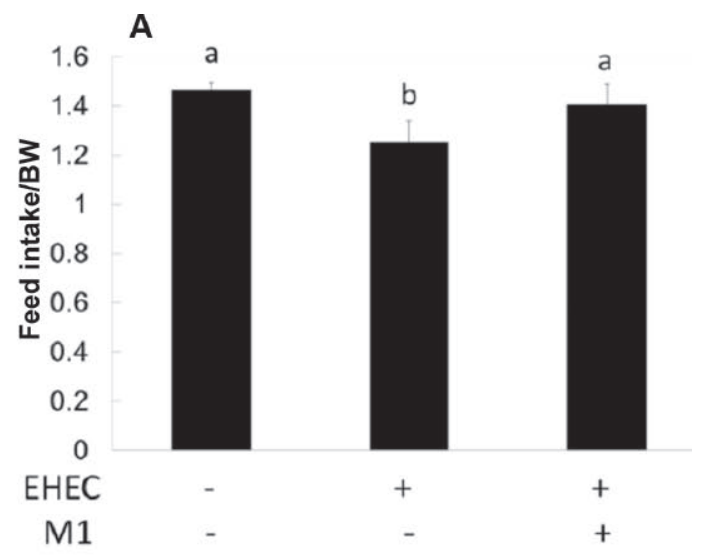

B
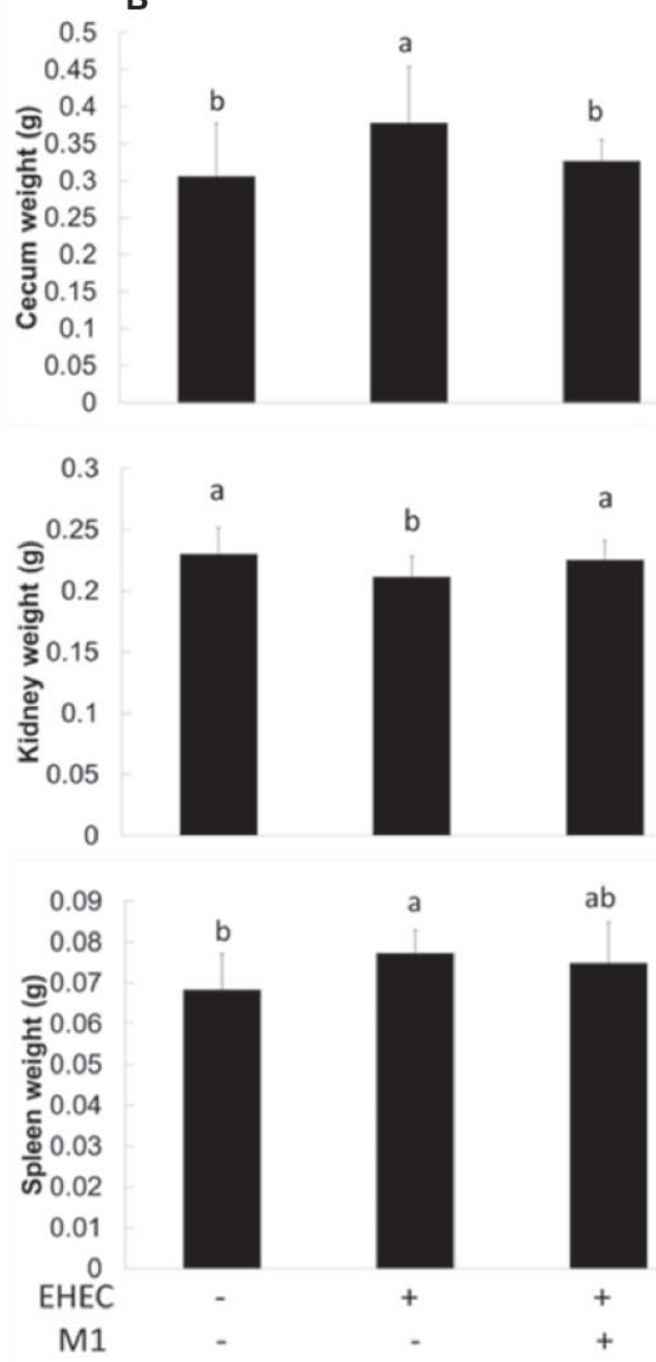

Figure 1. Effects of administration of Lactobacillus kefiranofaciens M1 on feed intake (A) and weight of collected organs (B) of enterohemorrhagic Escherichia coli (EHEC)-infected mice. Total feed intake per BW on d 16 was measured and calculated in each experimental group. The cecum, kidney, and spleen of each mouse in different treatment groups were collected and weighed after killing. Means for data without a common letter $(\mathrm{a}$ and $\mathrm{b})$ differ significantly $(P<0.05)$. The error bars represent the SD. to prevent the renal injury induced by EHEC infection in mice. In contrast, moderate congestion was observed in the heat-inactivated $L b$. kefiranofaciens M1-treated group (Figure 4), which shows that the protective ability of $L b$. kefiranofaciens M1 was reduced by heat treatment.

\section{Administration of Lb. kefiranofaciens M1 Decreases EHEC Translocation and Stx-1/Stx-2 Level in Serum}

Infection with EHEC may result in bacterial translocation across intestinal barrier and this can be followed by bacterial migration to the spleen (Figure $5 \mathrm{~A}$ ) and liver (Figure 5B) via the blood stream. Enterohemorrhagic $E$. coli translocation to both spleen and liver was significantly reduced in the Lb. kefiranofaciens M1-pretreated group compared with the EHEC-infected group (Figure 5). Surprisingly, no EHEC could be detected in the blood of the Lb. kefiranofaciens M1-pretreated group, whereas more than $10^{2} \mathrm{cfu} / \mathrm{mL}$ of EHEC were found in the blood of the EHEC-infected mice (data not shown).

The levels of the EHEC virulence factors, Stx- 1 and Stx-2, in serum and feces across the different treated groups were also examined. The results indicated that the EHEC-infected mice showed significantly higher Stx-1/Stx-2 levels in serum than both the nontreated negative control and the Lb. kefiranofaciens M1-pretreated group (Figure 6).

\section{Administration of Lb. kefiranofaciens M1 Increases Mucosal EHEC-Specific Immunoglobulin Response}

The mucosal and systemic production of immunoglobulin-specific to EHEC O157:H7 were assayed. We observed that the production of EHEC-specific IgA in feces (Figure 7), as well as EHEC-specific IgG and IgM in the serum (data not shown) were all significantly increased in both the EHEC-infected and Lb. kefiranofaciens M1-pretreated groups compared with the nonEHEC-treated control. Only the level of EHEC-specific fecal IgA in the Lb. kefiranofaciens M1-pretreated group was significantly higher than that of the EHEC-infected positive control (Figure 7). These findings indicate that pretreatment with $L b$. kefiranofaciens M1 seems to trigger mucosal $\mathrm{IgA}$ responses that help to protect against EHEC infection. In contrast, the production of systemic serum total IgA was not increased in the $L b$. kefiranofaciens M1-pretreated mice compared with the EHEC-infected positive control. The EHEC-infected mice showed a higher serum total IgA concentration than both the noninfected negative control and the $L b$. kefiranofaciens M1 pretreated groups (data not shown). 


\section{Control}

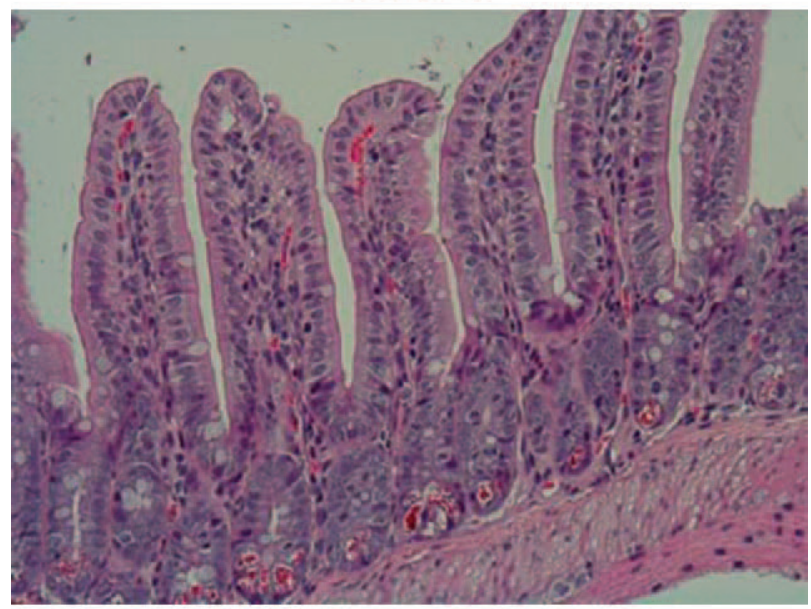

EHEC

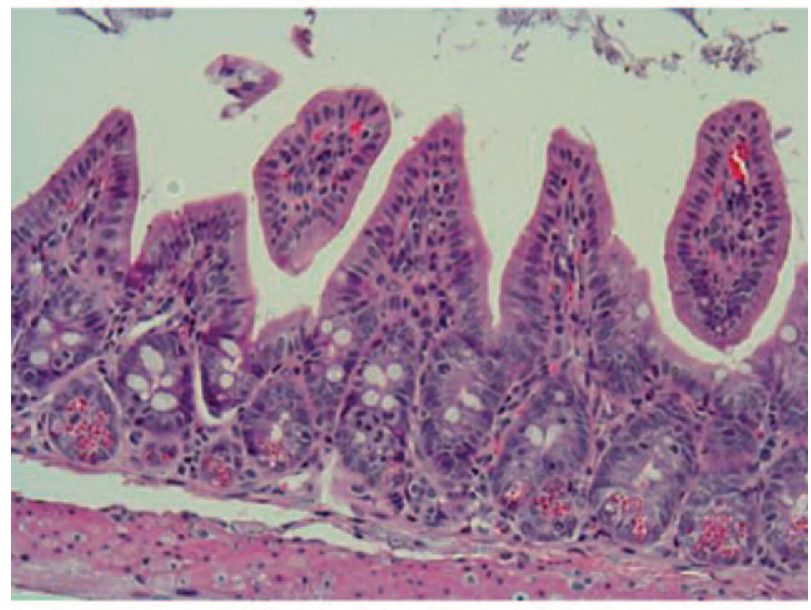

M1 + EHEC

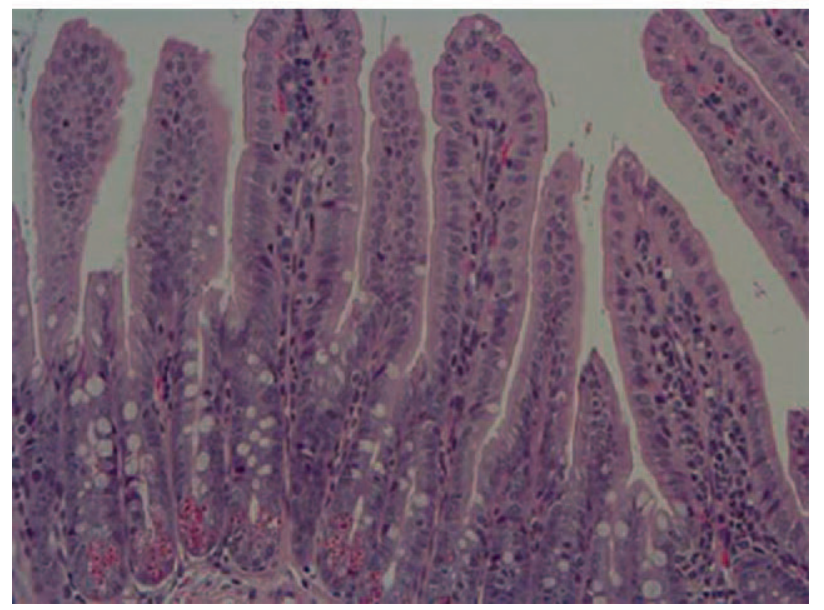

Figure 2. Effects of Lactobacillus kefiranofaciens M1 on histological evaluation of distal ileum sections of mice infected with enterohemorrhagic Escherichia coli (EHEC). Control = nontreated mice; EHEC $=$ EHEC-infected mice; $\mathrm{M} 1+\mathrm{EHEC}=$ mice pretreated with $L b$. kefiranofaciens M1 followed by EHEC infection. Color version available in the online PDF.

\section{Putative Mechanisms by Which Lb. kefiranofaciens M1 Can Protect Against EHEC In Vitro}

The protective effects of Lb. kefiranofaciens M1 on intestinal epithelial cells against EHEC-induced damage were investigated in vitro. We found that Lb. kefiranofaciens M1 was able to prevent intestinal epithelial cell Caco-2 death caused by EHEC treatment using both an MTT assay (Figure 8A) and an LDH assay (Figure $8 \mathrm{~B})$. In addition, the elicited protective effects were found to be significant in a dose-dependent manner when the ratio of $L b$. kefiranofaciens M1 to EHEC was 10:1 by both MTT assay (Figure 8A) and LDH assay (Figure 8B). This protective effect was not observed when heat-inactivated $L b$. kefiranofaciens M1 was used (Figures 8A and 8B). Furthermore, when we used a differentiated Caco-2 cell monolayer model to study the protective effects of $L b$. kefiranofaciens M1, the results showed that $L b$. kefiranofaciens M1 treatment was able to improve the EHEC-induced loss of Caco-2 monolayer integrity as measured by the TEER value (Figure 8C) and this effect was statistically significant $(P<0.05)$. On the other hand, Lb. kefiranofaciens M1 was not able to prevent the damage to the Caco-2 cell monolayer when the bacteria was cocultured with EHEC cell-free supernatant (Figure 9A). Furthermore, Lb. kefiranofaciens M1 also failed to reduce Stx levels in the EHEC supernatant (Figure 9B).

\section{DISCUSSION}

In the present study, we demonstrated a novel functionality for the kefir-isolated strain Lb. kefiranofaciens M1. After pretreatment with Lb. kefiranofaciens M1, all symptoms induced by EHEC infection, including fecal occult blood, reduction of feed intake (Figure 1), cecum enlargement, kidney atrophy, ileal villus atrophy (Figure 2), tissue edema, loss of epithelial integrity in the cecum (Figure 3A; Supplemental Figure S1, available online at http://dx.doi.org/10.3168/jds.20137015), and loss of epithelial integrity in the proximal colon (Figure 3B; Supplemental Figure S2), as well as renal injury (Figure 4), were improved in the mouse model. Similar findings have been reported for other lactobacilli. Lactobacillus rhamnosus HN001 (DR20) and Lactobacillus paracasei ssp. paracasei NTU 101 are able to reduce the severity of EHEC O157:H7 infection (Shu and Gill, 2002; Tsai et al., 2010). Lactobacillus reuteri ATCC PTA 6475 is able to ameliorate EHEC infection-mediated complications, such as renal tubular necrosis (Eaton et al., 2011). In terms of bifidobacteria, Bifidobacterium lactis HN019, Bifidobacterium breve strain Yakult, Bifidobacterium pseudocatenulatum DSM 20439, Bifidobacterium thermacidophilum RBL 71, Bi- 
A

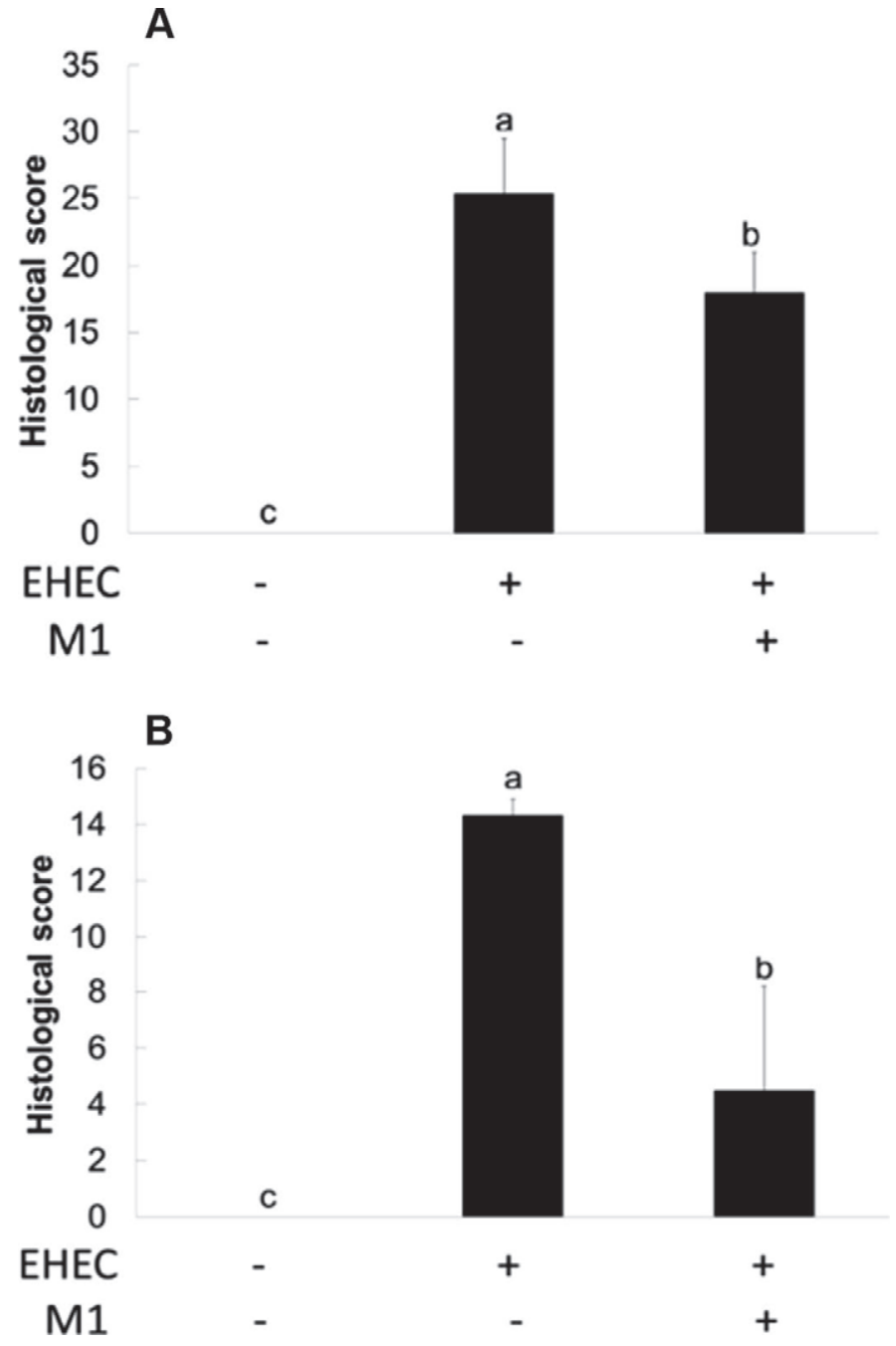

Figure 3. Effects of Lactobacillus kefiranofaciens M1 on quantitative histological scores of cecum (A) and colon (B) sections of mice infected with enterohemorrhagic Escherichia coli (EHEC). Histological scores were evaluated and means for data without a common letter $(\mathrm{a}-\mathrm{c})$ differ significantly $(P<0.05)$. Control $=$ nontreated mice; $\mathrm{EHEC}=$ EHEC-infected mice; $\mathrm{M} 1+\mathrm{EHEC}=$ mice pretreated with Lb. kefiranofaciens M1 followed by EHEC infection. The error bars represent the SD.

fidobacterium longum ssp. infantis $157 \mathrm{~F}-4-1$, B. longum ssp. longum NCC2705, and B. longum ssp. longum JCM $1217^{\mathrm{T}}$ have also been shown to have the ability to protect against EHEC O157:H7 infection. However, this was the first study to demonstrate that LAB from milk kefir has the anti-EHEC effect.

We further investigated the possible anti-EHEC mechanisms by which $L b$. kefiranofaciens M1 might act in vivo and in vitro. First, we demonstrated that EHEC translocation across the intestinal barrier and the bacterial migration to different organs via the bloodstream was significantly reduced in EHEC-infected mice pretreated with Lb. kefiranofaciens M1 (Figure 5). In addi- tion, the concentration of EHEC virulence factors Stx-1 and Stx2 in the serum was also significantly decreased (Figure 6). The reduction in EHEC and Stx translocation after treatment with $L b$. kefiranofaciens M1 might result from an enhancement of mucosal immunity via an elevation of the level of mucosal EHEC-specific IgA secretion in the EHEC-infected mice that had been pretreated with Lb. kefiranofaciens M1 (Figure 7). A T-celldependent high-affinity pathogen-specific IgA response has been reported to play an important role in protection against pathogen infection (Strugnell and Wijburg, 2010). The production of IgA has been found to be associated with activated toll-like receptor 2 (TLR2) and chemokine $\mathrm{C}-\mathrm{C}$ motif) ligand 20 (CCL20). The presence of TLR2 signaling adaptor protein MyD88 [myeloid differentiation primary response gene (88)] is necessary for the production of fecal IgA (Shang et al., 2008). Chemokine CCL20 and its receptor CCR6 pathway are required for localization of dendritic cells to the subepithelial dome of the Peyer's patch (PP); in addition, they have also been found to be responsible for the production of mucosal antigen-specific $\operatorname{IgA}$ in both the PP and lamina propia (Cook et al., 2000). In our previous study, Lb. kefiranofaciens M1 was found in vitro to increase production of chemokine CCL20 in both the apical and basolateral sides of an intestinal epithelial monolayer via TLR2 (Zhang et al., 2012). Both in vitro T helper cell 1 (Th1) cytokine induction and in vivo intestine protection of Lb. kefiranofaciens M1 have been shown to be dependent on TLR2 (Hong et al., 2009; Zhang et al., 2012). These findings suggest that the anti-EHEC mechanism of Lb. kefiranofaciens M1 is likely to be related to an increase in mucosal EHEC-specific IgA production via the TLR2/CCL20/ CCR6 pathway.

Another possible mechanism by which $L b$. kefiranofaciens M1 might elicit protection against EHEC infection is an enhancement of the functionality of the intestinal epithelial barrier (Figure 8) via TLR2. Tolllike receptor 2 is an important receptor that has been shown to be responsible for protecting the intestinal epithelial barrier against chemicals (Cario et al., 2007) and pathogen-induced colitis (Gibson et al., 2008). Several studies have reported that activation of TLR2 results in (1) enhancement of intestinal epithelial barrier integrity through redistribution of the tight junction protein ZO-1 by the protein kinase C (PKC) pathway (Cario et al., 2004; Gibson et al., 2008); (2) stimulation of the phosphatidylinositide 3-kinase/protein kinase B (PI3K/Akt) pathway, which induces antiapoptosis in intestinal epithelial cells (Cario et al., 2007); and (3) induction of goblet cell-derived trefoil factor (TFF)-3 production (Podolsky et al., 2009). Furthermore, TLR2 signaling adaptor protein MyD88 is also involved in 
protection against Stx-mediated disease that is caused by EHEC infection (Calderon Toledo et al., 2008).

Additionally, in our previous study, Lb. kefiranofaciens M1 was demonstrated to have an immunityenhancing ability that occurred by activating macrophages beneath the $\mathrm{PP}$, which, in turn, induced production of Th1 cytokines [IL-1 $\beta$, IL-6, IL-12, and tumor necrosis factor $\alpha$ (TNF- $\alpha)$; Hong et al., 2009] via TLR2. Etienne-Mesmin et al. (2011) reported that EHEC is able to translocate into the body through $\mathrm{M}$ cells to the PP in mice, where it might be further endocytosed by macrophages. The engulfed EHEC is able to survive in macrophages and this induces macrophage apoptosis in an Stx-2-dependent way, which is followed by release of the Stx, which results in toxicity (Etienne-Mesmin et al., 2011). Therefore, enhancement of the bactericidal activity of macrophages is important to the inhibition of EHEC infection. The bactericidal activity may be enhanced by an increase in the level of cellular hydrogen peroxide via activation of the TLR signaling pathway, including TLR2 (West et al., 2011). Activation of macrophages via TLR2 might be one of the mechanisms by which Lb. kefiranofaciens M1 acts against EHEC infection.

Several studies have investigated the possible mechanisms by which lactic acid bacteria elicit protection against EHEC infection. Johnson-Henry et al. (2008) indicated that $L b$. rhamnosus GG is able to prevent EHEC-induced barrier function loss in vitro by restoring tight junction protein ZO-1 and claudin-1 redistribution, which are induced by EHEC. Putaala et al. (2008) has shown that the barrier function-enhancing strain B. lactis 420 is able to block intestinal barrier damage that is induced by Stx secreted by EHEC. Lactobacillus rhamnosus HN001 (DR20TM) and Lb. paracasei ssp. paracasei NTU 101 are able to reduce the severity of EHEC O157:H7 infection by enhancing immune cell function and immunoglobulin production (Shu and Gill, 2002; Tsai et al., 2010). In addition, some studies also reported that certain strains are able to directly eliminate Stx-derived toxicity of intestinal epithelial cells monolayers; this is thought to happen via the binding of Stx by cell lysate, which neutralizes Stx toxicity (Nishikawa et al., 2002). However, we found that Lb. kefiranofaciens M1 was not able to remove Stx directly (Figure 9), suggesting that this mode of action is not applicable to Lb. kefiranofaciens M1.

Above all, administration of Lb. kefiranofaciens M1 was found in this study to be able to prevent or reduce the severity of EHEC infection, which fits the generally accepted concept that inhibition of initial EHEC infection is the best method of preventing EHEC infection and the hemolytic uremic syndrome it mediates (Serna and Boedeker, 2008). Possible mechanisms include the

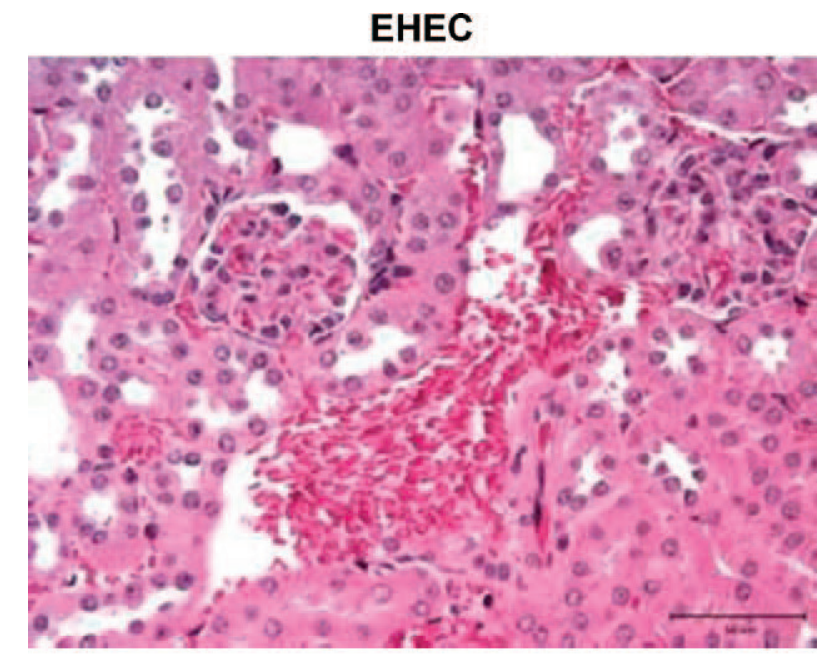

\section{M1 + EHEC}

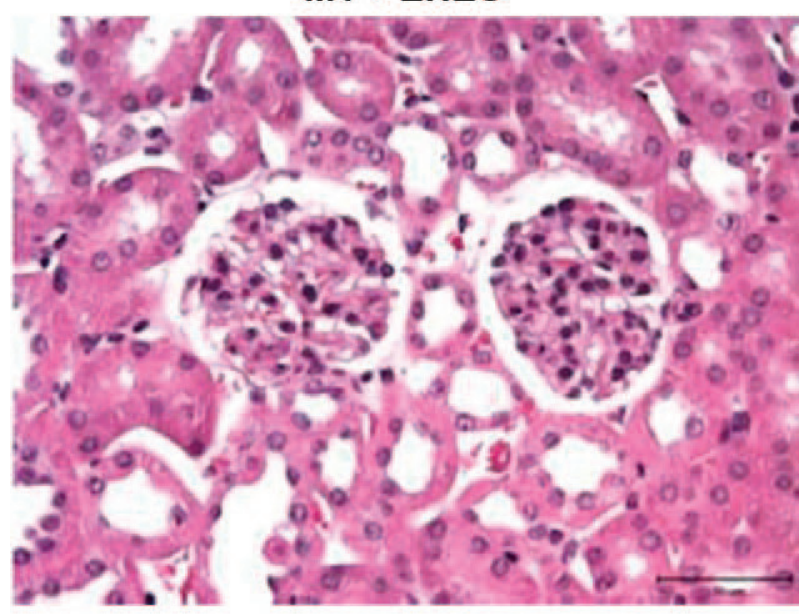

HIM1 + EHEC

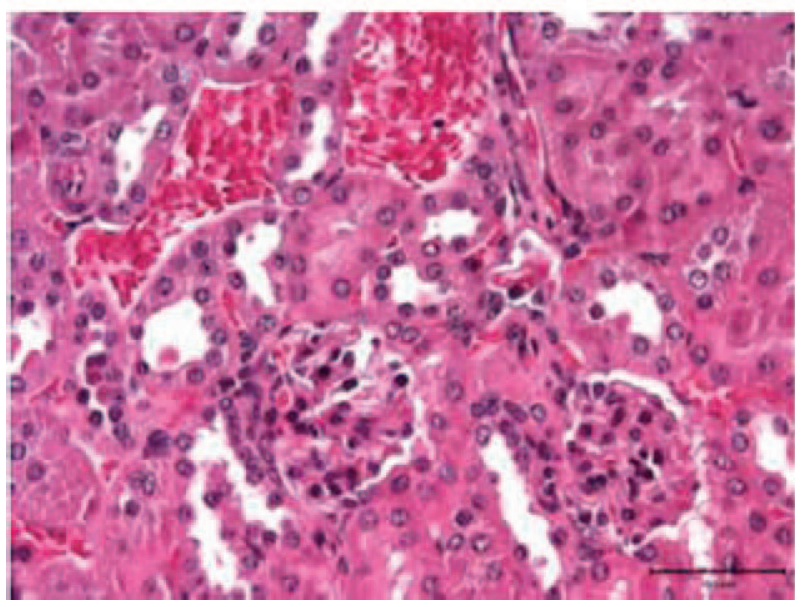

Figure 4. Effects of Lactobacillus kefiranofaciens M1 on histological evaluation of kidney sections of mice infected with enterohemorrhagic Escherichia coli (EHEC). EHEC = EHEC-infected mice; M1 + EHEC $=$ mice pretreated with $L b$. kefiranofaciens M1 followed by EHEC infection; HIM1 + EHEC = mice pretreated with heat-inactivated $L b$. kefiranofaciens M1 followed by EHEC infection. Color version available in the online PDF. 

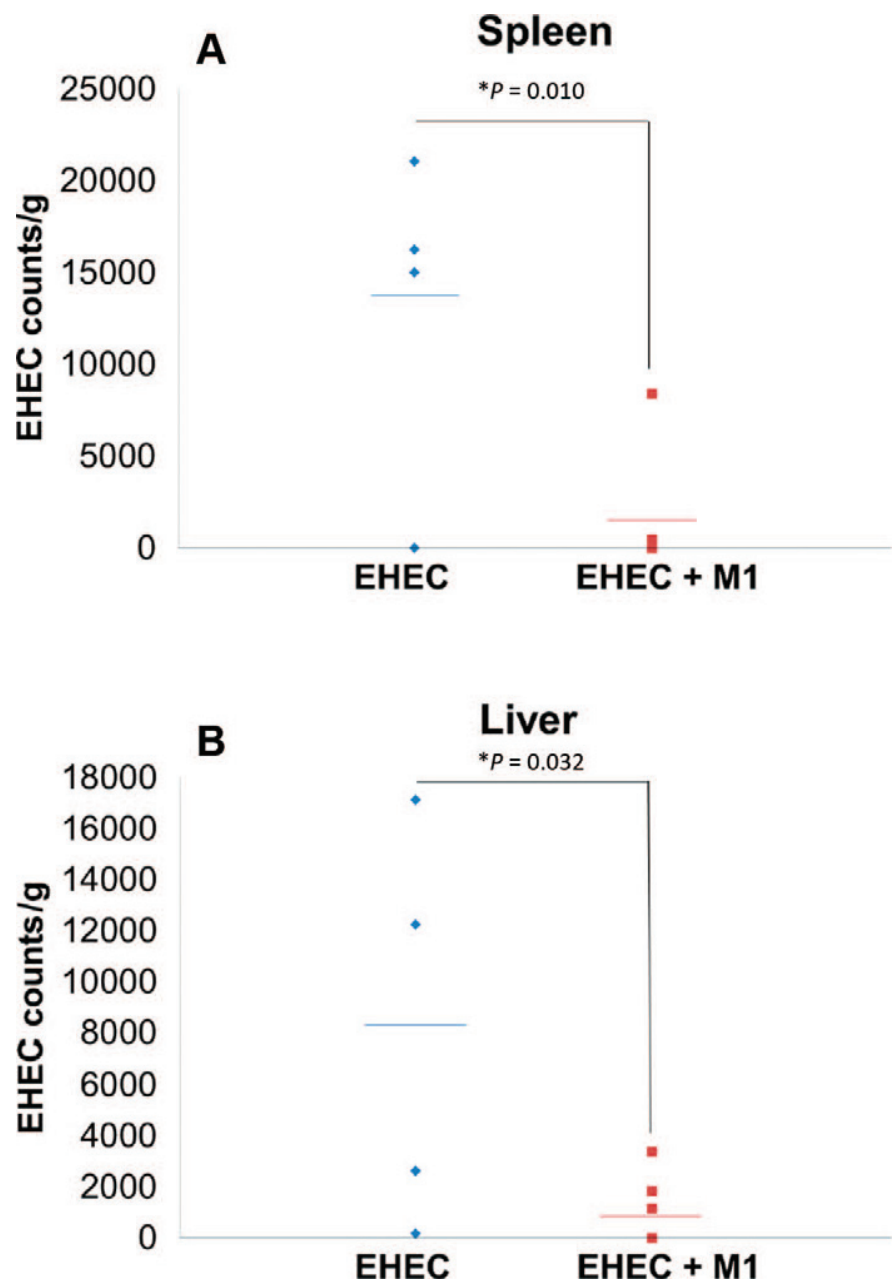

Figure 5. Administration of Lactobacillus kefiranofaciens M1 reduces enterohemorrhagic Escherichia coli (EHEC) translocation to the spleen and liver in mice. The EHEC in organs were counted by using CHROMagar O157 (CHROMagar, Paris, France) after homogenization. $P$-values were calculated by Student's $t$-test. EHEC $=$ EHECinfected mice $(\mathrm{n}=4) ; \mathrm{M} 1+\mathrm{EHEC}=$ mice pretreated with $\mathrm{Lb}$. Kefiranofaciens M1 followed by EHEC infection $(\mathrm{n}=6)$. Color version available in the online PDF.

enhancement of mucosal immunity and improvements in intestinal barrier functionality, both of which will result in the reduction of EHEC translocation to other organs and a decrease in the concentration of EHEC virulence factors in the serum. Lactobacillus kefiranofaciens M1 seems to activate macrophages to produce Th1 cytokines and these may also play an important role in pathogen infection defense. Finally, the ability of $L b$. kefiranofaciens M1 to enhance mucosal immunity is indicative that $L b$. kefiranofaciens M1 might also be useful as a method of preventing other enteric pathogen infections. These findings might also provide scientific evidence for certain health benefits of milk kefir products.

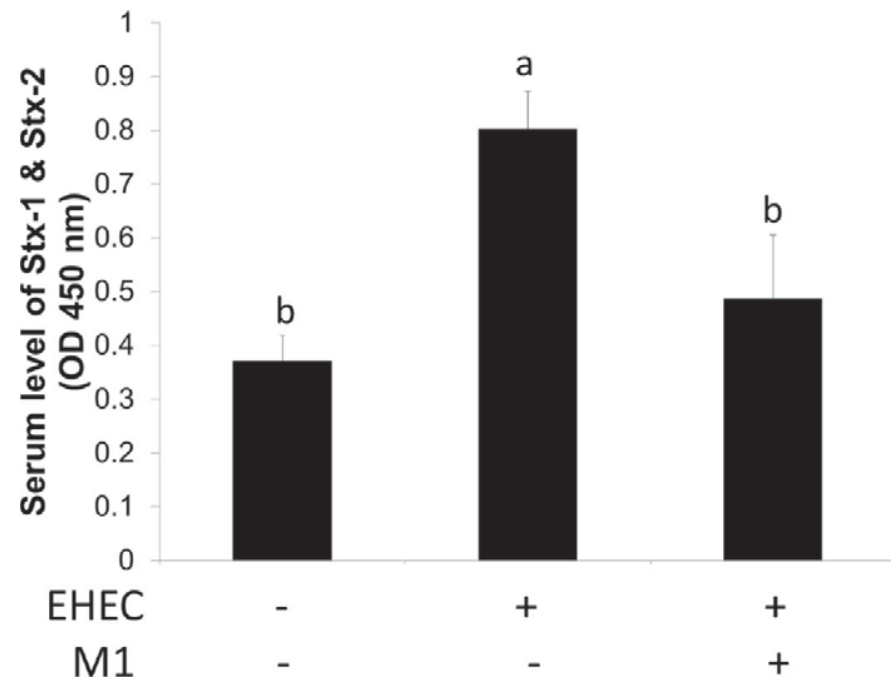

Figure 6. Administration of Lactobacillus kefiranofaciens M1 (M1) decreases serum Shiga toxin-1 and -2 (Stx- 1 and Stx-2) levels in enterohemorrhagic Escherichia coli (EHEC)-infected mice. Means for data without a common letter (a and b) differ significantly $(P<0.05)$. $\mathrm{OD}=$ optical density. The error bars represent the SD.

\section{ACKNOWLEDGMENTS}

We thank the National Science Council (Taipei, Taiwan) for grant support. We thank Je-Ruei Liu (Institute of Biotechnology, National Taiwan University, Taipei, Taiwan) for providing EHEC and critical discussion. We thank Chian-Ren Jeng and Yi-Chun Liao (Graduate Institute of Molecular and Compara-

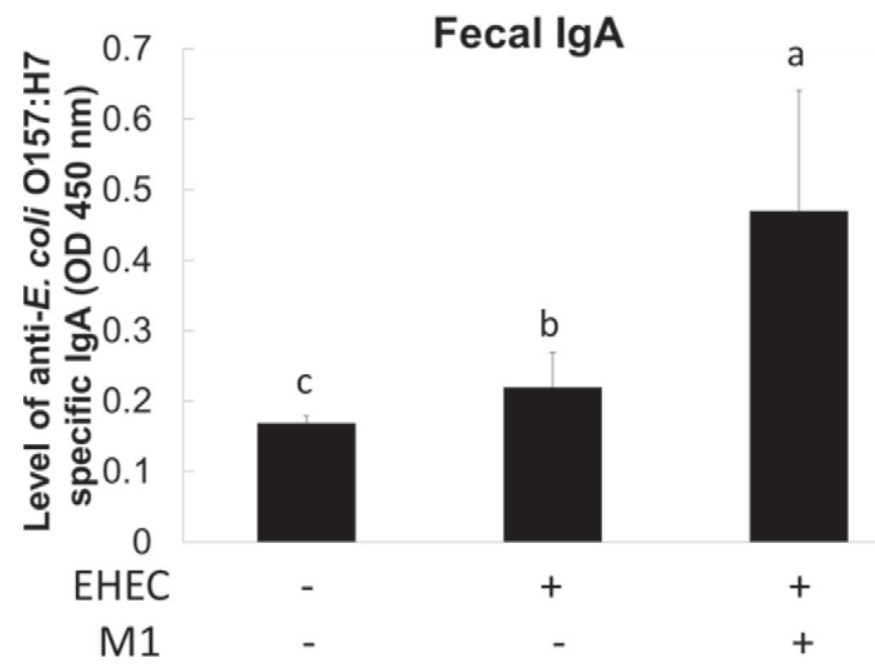

Figure 7. Effects of administration with Lactobacillus kefiranofaciens M1 (M1) on anti-Escherichia coli O157:H7-specific immunoglobulin production in enterohemorrhagic Escherichia coli (EHEC)infected mice. Fecal anti-E. coli O157:H7-specific IgA from different treated groups were measured. Means for data without a common letter $(\mathrm{a}-\mathrm{c})$ differ significantly $(P<0.05)$. $\mathrm{OD}=$ optical density. The error bars represent the SD. 
A
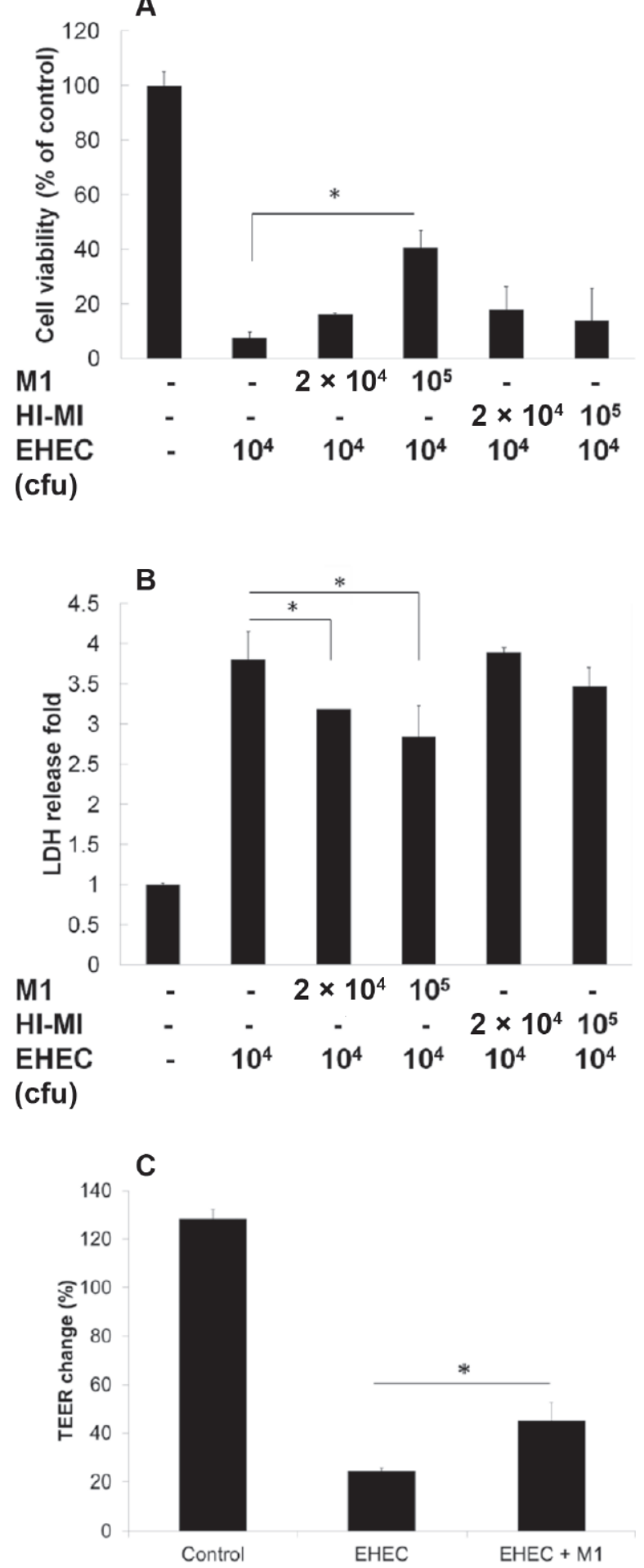

Figure 8. Lactobacillus kefiranofaciens M1 (M1) protects intestinal epithelial cell Caco-2 against enterohemorrhagic Escherichia coli (EHEC)-induced cell death and monolayer integrity in vitro. Cell viability was evaluated by (A) 3-(4,5-dimethyl-2-thiazolyl)-2,5diphenyl-2H-tetrazolium bromide (MTT) and (B) lactose dehydrogenase $(\mathrm{LDH})$ methods. Intestinal epithelial cell monolayer integrity was assayed by measurement of the transepithelial electric resistance (TEER; C). HI-M1 = heat-inactivated Lb. kefiranofaciens $\mathrm{M} 1 .{ }^{*} P<$ 0.05. The error bars represent the SD.
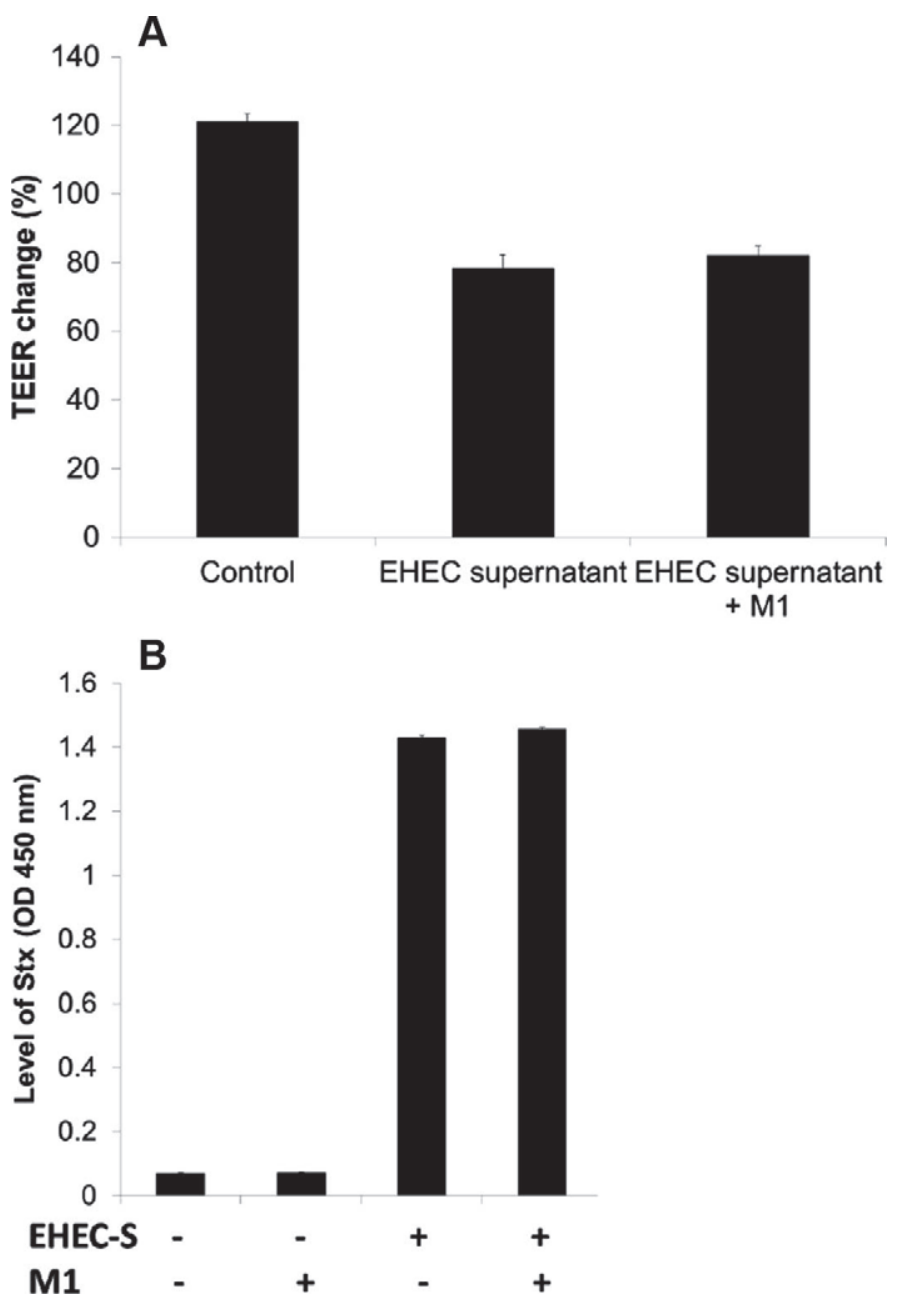

Figure 9. Effects of Lactobacillus kefiranofaciens M1 (M1) on enterohemorrhagic Escherichia coli cultured supernatant (EHEC-S). (A) Effect of Lb. kefiranofaciens M1 on EHEC-S-induced damage to intestinal epithelial monolayer was evaluated by measuring the loss of transepithelial electric resistance (TEER) of the Caco-2 monolayer. The EHEC-S was added $20 \%$ (vol/vol) in Caco-2 monolayer cell medium. (B) The effect of Lb. kefiranofaciens M1 on the level of Shiga-like toxins (Stx) in EHEC-S was measured by ELISA. OD = optical density. The error bars represent the SD.

tive Pathology, School of Veterinary Medicine, National Taiwan University) for help of histological evaluations. We thank Cheng-Yi Kuo (Foreway Biotechnology Inc., Taipei Hsien, Taiwan) for modification of illustrations.

\section{REFERENCES}

Calderon Toledo, C. C., T. J. Rogers, M. Svensson, R. Tati, H. Fischer, C. Svanborg, and D. Karpman. 2008. Shiga toxin-mediated disease in MyD88-deficient mice infected with Escherichia coli O157:H7. Am. J. Pathol. 173:1428-1439

Cario, E., G. Gerken, and D. K. Podolsky. 2004. Toll-like receptor 2 enhances ZO-1-associated intestinal epithelial barrier integrity via protein kinase C. Gastroenterology 127:224-238.

Cario, E., G. Gerken, and D. K. Podolsky. 2007. Toll-like receptor 2 controls mucosal inflammation by regulating epithelial barrier function. Gastroenterology 132:1359-1374. 
Chen, H.-C., S.-Y. Wang, and M.-J. Chen. 2008. Microbiological study of lactic acid bacteria in kefir grains by culture-dependent and culture-independent methods. Food Microbiol. 25:492-501.

Chen, Y. P., P. J. Hsiao, W. S. Hong, T. Y. Dai, and M. J. Chen. 2012. Lactobacillus kefiranofaciens M1 isolated from milk kefir grains ameliorates experimental colitis in vitro and in vivo. J. Dairy Sci. 95:63-74.

Cook, D. N., D. M. Prosser, R. Forster, J. Zhang, N. A. Kuklin, S. J. Abbondanzo, X.-D. Niu, S.-C. Chen, D. J. Manfra, M. T. Wiekowski, L. M. Sullivan, S. R. Smith, H. B. Greenberg, S. K. Narula, M. Lipp, and S. A. Lira. 2000. CCR6 mediates dendritic cell localization, lymphocyte homeostasis, and immune responses in mucosal tissue. Immunity 12:495-503.

Dieleman, L. A., M. J. H. J. Palmen, H. Akol, E. Bloemena, A. S. Peña, S. G. M. Meuwissen, and E. P. Van Rees. 1998. Chronic experimental colitis induced by dextran sulphate sodium (DSS) is characterized by Th1 and Th2 cytokines. Clin. Exp. Immunol. 114:385-391.

DuPont, A. W., and H. L. DuPont. 2011. The intestinal microbiota and chronic disorders of the gut. Nat. Rev. Gastroenterol. Hepatol. 8:523-531.

Eaton, K. A., A. Honkala, T. A. Auchtung, and R. A. Britton. 2011. Probiotic Lactobacillus reuteri ameliorates disease due to enterohemorrhagic Escherichia coli in germfree mice. Infect. Immun. 79:185-191.

Etienne-Mesmin, L., B. Chassaing, P. Sauvanet, J. Denizot, S. Blanquet-Diot, A. Darfeuille-Michaud, N. Pradel, and V. Livrelli. 2011. Interactions with $\mathrm{M}$ cells and macrophages as key steps in the pathogenesis of enterohemorragic Escherichia coli infections. PLoS ONE 6:e23594.

Gagnon, M., E. E. Kheadr, N. Dabour, D. Richard, and I. Fliss. 2006. Effects of Bifidobacterium thermacidophilum probiotic feeding on enterohemorrhagic Escherichia coli O157:H7 infection in BALB/c mice. Int. J. Food Microbiol. 111:26-33.

Gareau, M. G., P. M. Sherman, and W. A. Walker. 2010. Probiotics and the gut microbiota in intestinal health and disease. Nat. Rev. Gastroenterol. Hepatol. 7:503-514.

Gibson, D. L., C. Ma, C. M. Rosenberger, K. S. B. Bergstrom, Y. Valdez, J. T. Huang, M. A. Khan, and B. A. Vallance. 2008. Tolllike receptor 2 plays a critical role in maintaining mucosal integrity during Citrobacter rodentium-induced colitis. Cell. Microbiol. 10:388-403.

Guzel-Seydim, Z. B., T. Kok-Tas, A. K. Greene, and A. C. Seydim. 2011. Review: Functional properties of kefir. Crit. Rev. Food Sci. Nutr. 51:261-268.

Hodges, K., and R. Gill. 2010. Infectious diarrhea: Cellular and molecular mechanisms. Gut Microbes 1:4-21.

Hong, W.-S., H.-C. Chen, Y.-P. Chen, and M.-J. Chen. 2009. Effects of kefir supernatant and lactic acid bacteria isolated from kefir grain on cytokine production by macrophage. Int. Dairy J. 19:244-251.

Hong, W.-S., Y.-P. Chen, and M.-J. Chen. 2010. The antiallergic effect of kefir lactobacilli. J. Food Sci. 75:H244-H253.

Hong, W.-S., Y.-P. Chen, T.-Y. Dai, I.-N. Huang, and M.-J. Chen. 2011. Effect of heat-inactivated kefir-isolated Lactobacillus kefiranofaciens $\mathrm{M} 1$ on preventing an allergic airway response in mice. J. Agric. Food Chem. 59:9022-9031.

Johnson-Henry, K. C., K. A. Donato, G. Shen-Tu, M. Gordanpour, and P. M. Sherman. 2008. Lactobacillus rhamnosus strain GG prevents enterohemorrhagic Escherichia coli O157:H7-induced changes in epithelial barrier function. Infect. Immun. 76:1340-1348.

Kaper, J. B., J. P. Nataro, and H. L. T. Mobley. 2004. Pathogenic Escherichia coli. Nat. Rev. Microbiol. 2:123-140.
Lim, J. Y., J. W. Yoon, and C. J. Hovde. 2010. A brief overview of Escherichia coli O157:H7 and its plasmid O157. J. Microbiol. Biotechnol. 20:5-14.

Mohawk, K. L., A. R. Melton-Celsa, T. Zangari, E. E. Carroll, and A. D. O'Brien. 2010. Pathogenesis of Escherichia coli O157:H7 strain 86-24 following oral infection of BALB/c mice with an intact commensal flora. Microb. Pathog. 48:131-142.

Mohawk, K. L., and A. D. O'Brien. 2011. Mouse models of Escherichia coli O157:H7 infection and Shiga toxin injection. J. Biomed. Biotechnol. 2011:258185.

Nishikawa, K., K. Matsuoka, E. Kita, N. Okabe, M. Mizuguchi, K. Hino, S. Miyazawa, C. Yamasaki, J. Aoki, S. Takashima, Y. Yamakawa, M. Nishijima, D. Terunuma, H. Kuzuhara, and Y. Natori. 2002. A therapeutic agent with oriented carbohydrates for treatment of infections by Shiga toxin-producing Escherichia coli O157:H7. Proc. Natl. Acad. Sci. USA 99:7669-7674.

Podolsky, D. K., G. Gerken, A. Eyking, and E. Cairo. 2009. Colitis-associated variant of TLR2 causes impaired mucosal repair because of TFF3 deficiency. Gastroenterology 137:209-220.

Putaala, H., T. Salusjärvi, M. Nordström, M. Saarinen, A. C. Ouwehand, E. B. Hansen, and N. Rautonen. 2008. Effect of four probiotic strains and Escherichia coli O157:H7 on tight junction integrity and cyclo-oxygenase expression. Res. Microbiol. 159:692-698.

Santos, A., M. San Mauro, A. Sanchez, J. M. Torres, and D. Marquina. 2003. The antimicrobial properties of different strains of Lactobacillus spp. isolated from kefir. Syst. Appl. Microbiol. 26:434-437.

Serna, A., IV, and E. C. Boedeker. 2008. Pathogenesis and treatment of Shiga toxin-producing Escherichia coli infections. Curr. Opin. Gastroenterol. 24:38-47.

Shang, L., M. Fukata, N. Thirunarayanan, A. P. Martin, P. Arnaboldi, D. Maussang, C. Berin, J. C. Unkeless, L. Mayer, M. T. Abreu, and S. A. Lira. 2008. TLR signaling in small intestinal epithelium promotes B cell recruitment and IgA production in lamina propria. Gastroenterology 135:529-538.

Shu, Q., and H. S. Gill. 2002. Immune protection mediated by the probiotic Lactobacillus rhamnosus HN001 (DR20 ${ }^{\mathrm{TM}}$ ) against Escherichia coli O157:H7 infection in mice. FEMS Immunol. Med. Microbiol. 34:59-64.

Strugnell, R. A., and O. L. C. Wijburg. 2010. The role of secretory antibodies in infection immunity. Nat. Rev. Microbiol. 8:656-667.

Tarr, P. I., C. A. Gordon, and W. L. Chandler. 2005. Shiga-toxin-producing Escherichia coli and haemolytic uraemic syndrome. Lancet 365:1073-1086.

Tsai, Y.-T., P.-C. Cheng, and T.-M. Pan. 2010. Immunomodulating activity of Lactobacillus paracasei subsp. paracasei NTU 101 in enterohemorrhagic Escherichia coli O157H7-infected mice. J. Agric. Food Chem. 58:11265-11272.

Wang, S. Y., H. C. Chen, J. R. Liu, Y. C. Lin, and M. J. Chen. 2008. Identification of yeasts and evaluation of their distribution in Taiwanese kefir and viili starters. J. Dairy Sci. 91:3798-3805.

West, A. P., I. E. Brodsky, C. Rahner, D. K. Woo, H. ErdjumentBromage, P. Tempst, M. C. Walsh, Y. Choi, G. S. Shadel, and S. Ghosh. 2011. TLR signaling augments macrophage bactericidal activity through mitochondrial ROS. Nature 472:476-480.

Zhang, X., Y. Cheng, Y. Xiong, C. Ye, H. Zheng, H. Sun, H. Zhao, Z. Ren, and J. Xu. 2012. Enterohemorrhagic Escherichia coli specific enterohemolysin induced IL-1 $\beta$ in human macrophages and EHEC-induced IL-1 $\beta$ required activation of NLRP3 inflammasome. PLoS ONE 7:e50288. 